Journal of Sustainable Agricultural Sciences
http://jsas.journals.ekb.eg/

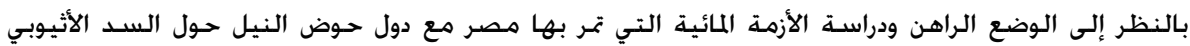

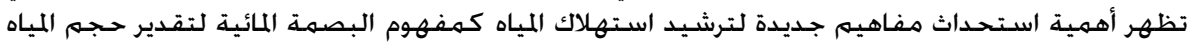

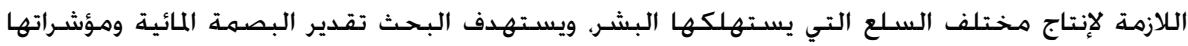

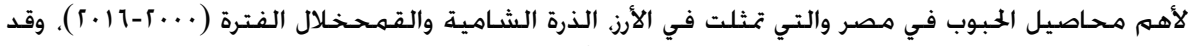

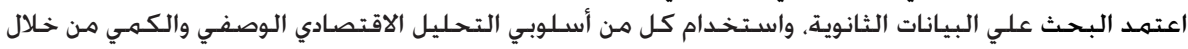

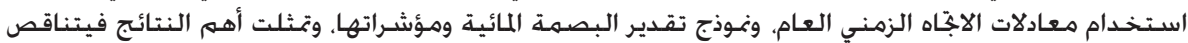

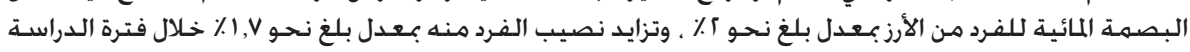

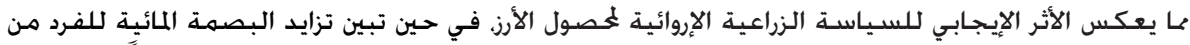

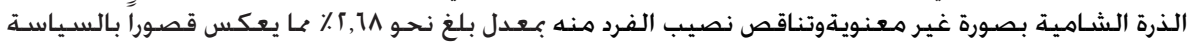

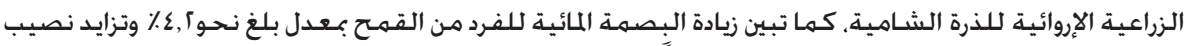

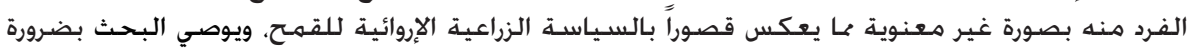

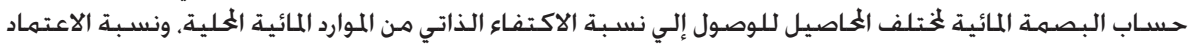

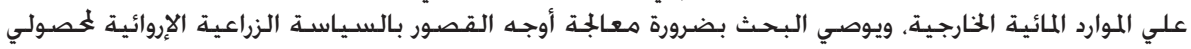

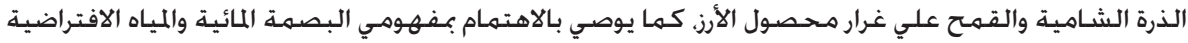
بما يحقق الكفاءة في استخـدام مورد المياه.

الكلمات الافتتاحية: البصمة المائية - متوسط نصيب الفرد من الغصصول - نسبة الاكتفاء الذاتي من الموارد المائية.

وحتي وصولها ليد المستهلك النهائي ومفهوم البصمة المائية لا

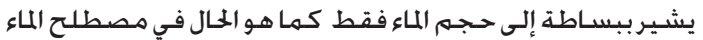

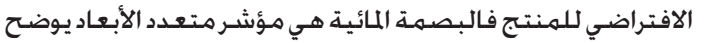

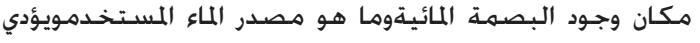

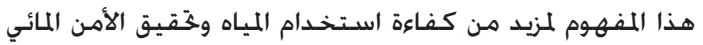
في المناطق الجافة.

المشكلة البحثية

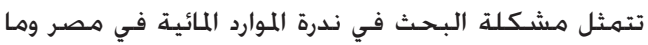

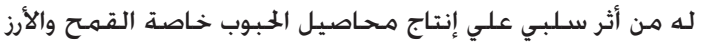

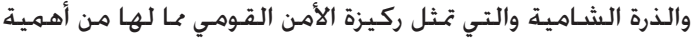

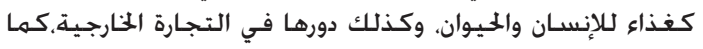

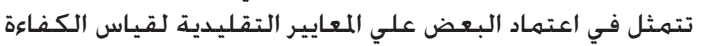

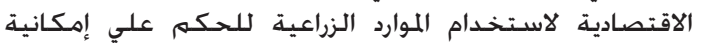

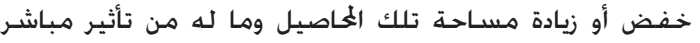

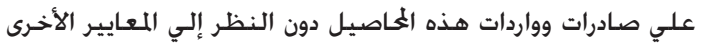

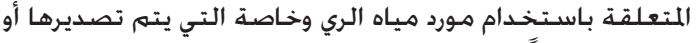

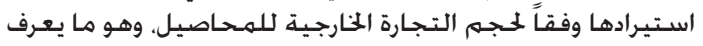

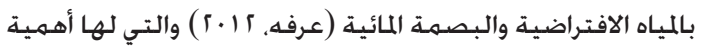

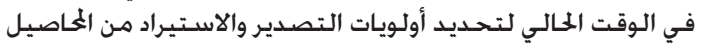

مقدمة

تعتبر المياهمن القضايا الههامة والحيوية لتحقيق التنمية الاقتصادية

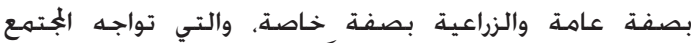

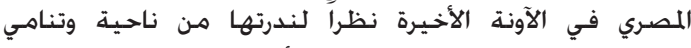

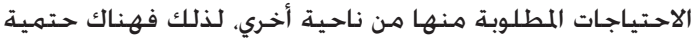

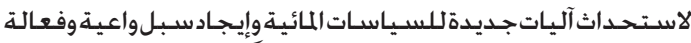

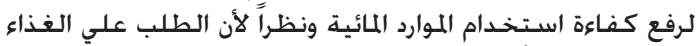

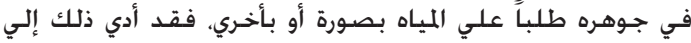

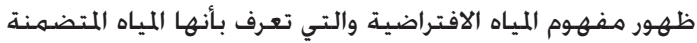

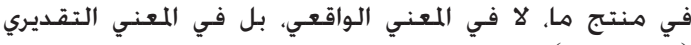

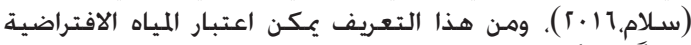

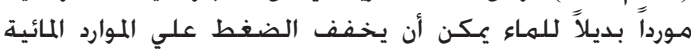

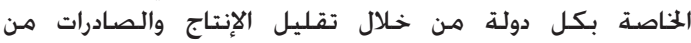

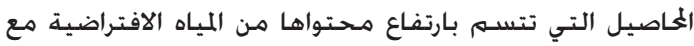

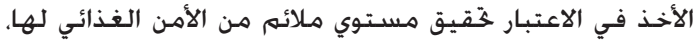

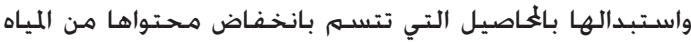

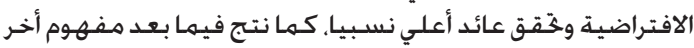

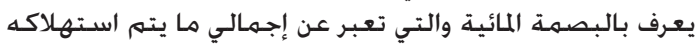

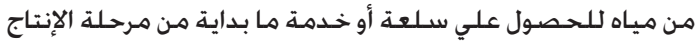

*Corresponding author: mo2455599@gmail.com

DOI : $10.21608 /$ jsas.2019.17078.1168

Received:18/9/2019; Accepted:7/11/2019

C2019 National Information and Documentation Center (NIDOC) 
الدولية للأغذية أن يكون التبادل التجاري بين الدول سـياسـة

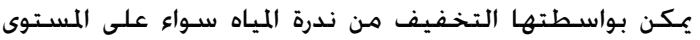

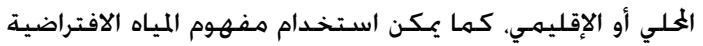

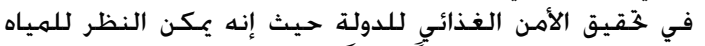

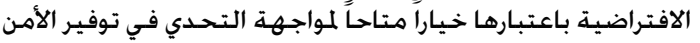

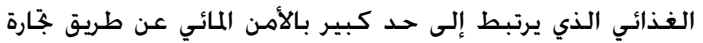

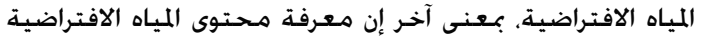

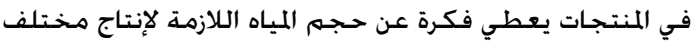

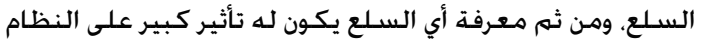
المائي وكيف يمكن خحقيق وفر مائي من خلال ذلك الك.

نموذج تقدير البصمة المائية ومؤشراتها (سـحر قمره، ع ( • ؟): يستند تقدير البصمة المائية علي البيانات التالية:

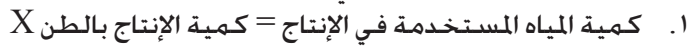
الاحتياجات المائية للطن.

r. كمية المياه الافتراضية المصدرة = كمية الصادرات بالطن الاحتياجات المائية للطن.

r. كمية المياه الافتراضية المستوردة = كمية الواردات بالطن الاحتياجات المائية للطن.

ويته حسـاب البصمة المائية كما يلي:

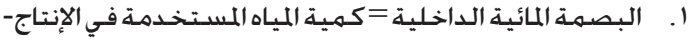
كمية المياه الافتراضية المصدرة. ז. البصمة المائية الخارجية = كمية المياه الافتراضية

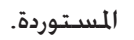

r. البصمة المائية الكلية= البصمة المائية الداخلية+ البصمة المائية الخارجية

وتتمثل مؤشـرات البصمة المائية فيما يلي:

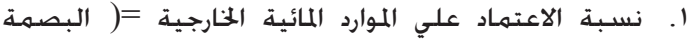

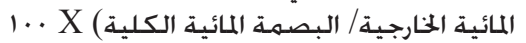

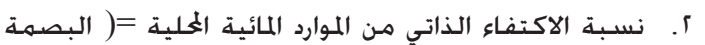

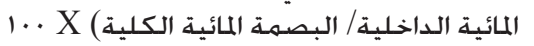

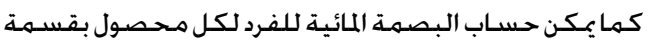

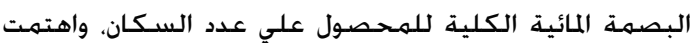

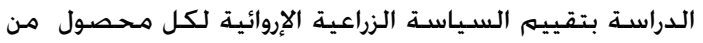

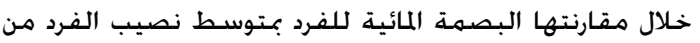

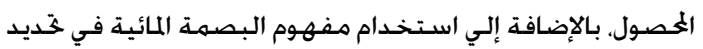
أولويات التجارة الخارجية من صالإدية استخدات وواردات.

النتائج البحثية

أولاً: البصمة المائية والسياسـة الزراعية الإروائية لأهم محاصيل الحبوب: البه

حيث تعتبر محاصيل الأرز، الذرة الشـامية والقـمـح من أهم الهم

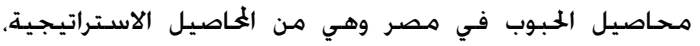

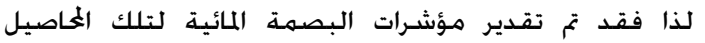

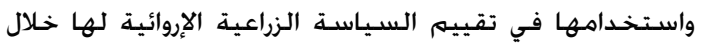

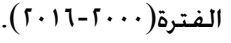

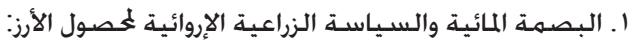

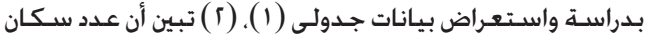

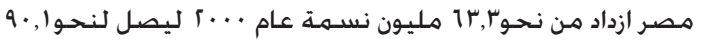

موضع الدراسـة لنحقيق الأمن الغذائي من ناحية، وترشيد استخـدام مياه الري من ناحية أخري.

الأهداف البحثبة

يســهـدف البحـث تقدير كفـاءة اسـتخـدام الموارد المائية الإروائية من خـلال ما يلي:

ا. تقـدير أهم مؤشـرات المياه الافتراضية والبصمة المائية

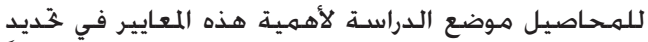

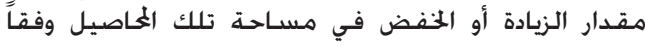

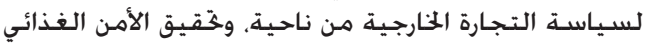
من هذه العاصيل من ناحية أخـري.

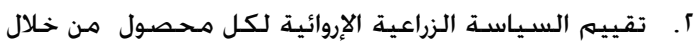

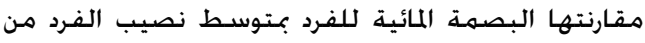
المـصول. مصادر البيانات والأسلوب البحثي

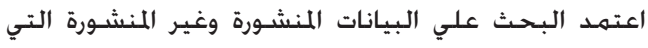

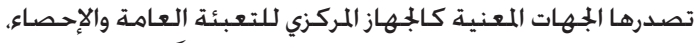

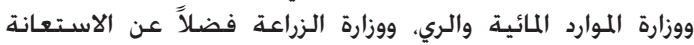

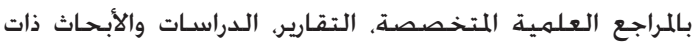

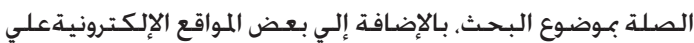
شبكـة المعلومات الدولية.

كـما اعتمـد البــث في ختقيق أهدافه علي كل مـن أسـلوبي

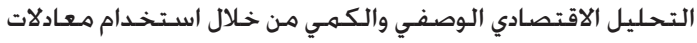

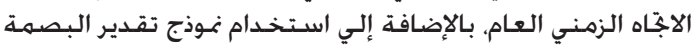

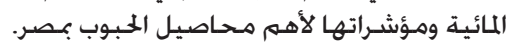

البصمة المائية Water footprint تعرف البصمة المائية وفقا لموقع شبكة البــة البصمة المائية بأنها

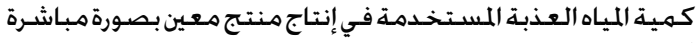

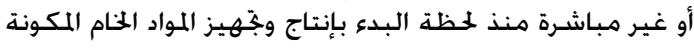

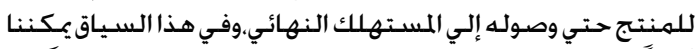

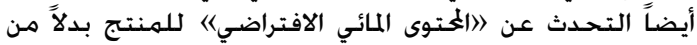

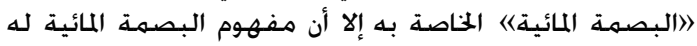

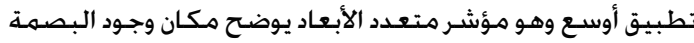

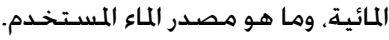

البصمـة المائية الكلية للدولة:

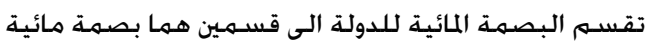

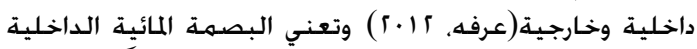

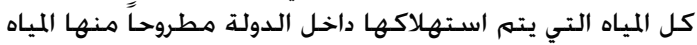

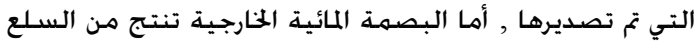

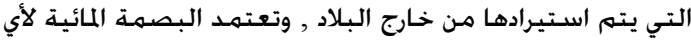

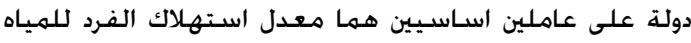
وكفاءة نظام إدارة الموارد المائية في تلك املك الدولة .

أهـميـة دراسـة المياه الافتراضيـة:

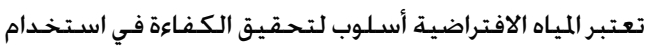

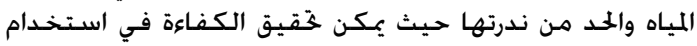

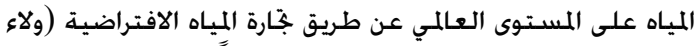

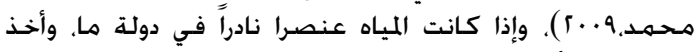

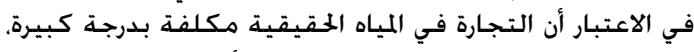

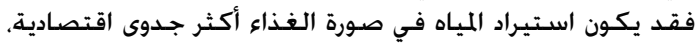
وفي ضوء ذلك اقترح عدد كبير من الباحثين في مجال التجارة

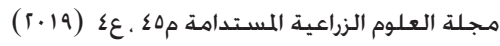




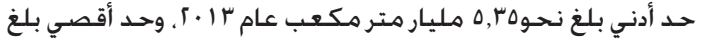

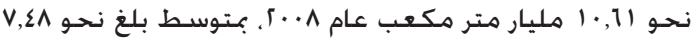
مليار متر مكعب.

كما تبين أن نسبـة الاعتماد علي الموارد المائية الخارجية

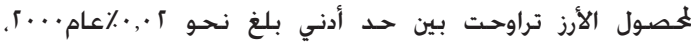

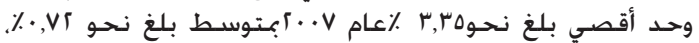

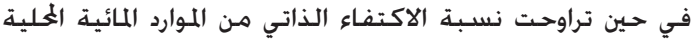

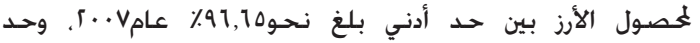

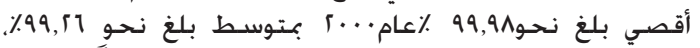

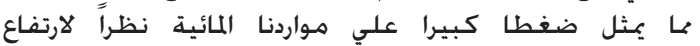
الاحتياجـات المائية للأرز.

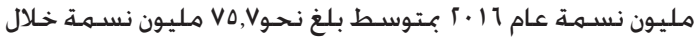

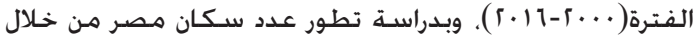

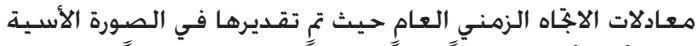

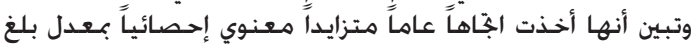

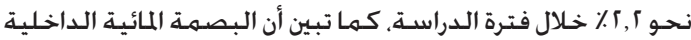

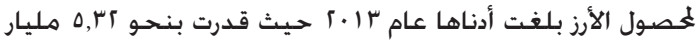

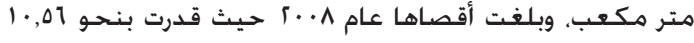

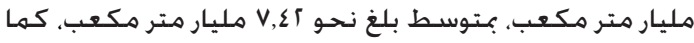

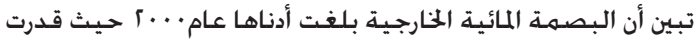

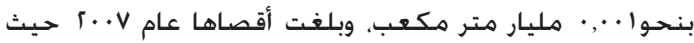

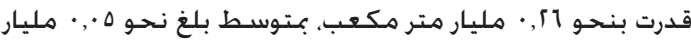

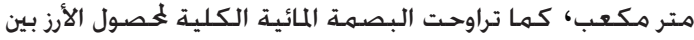

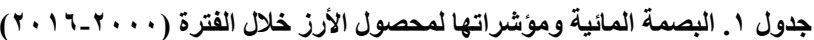

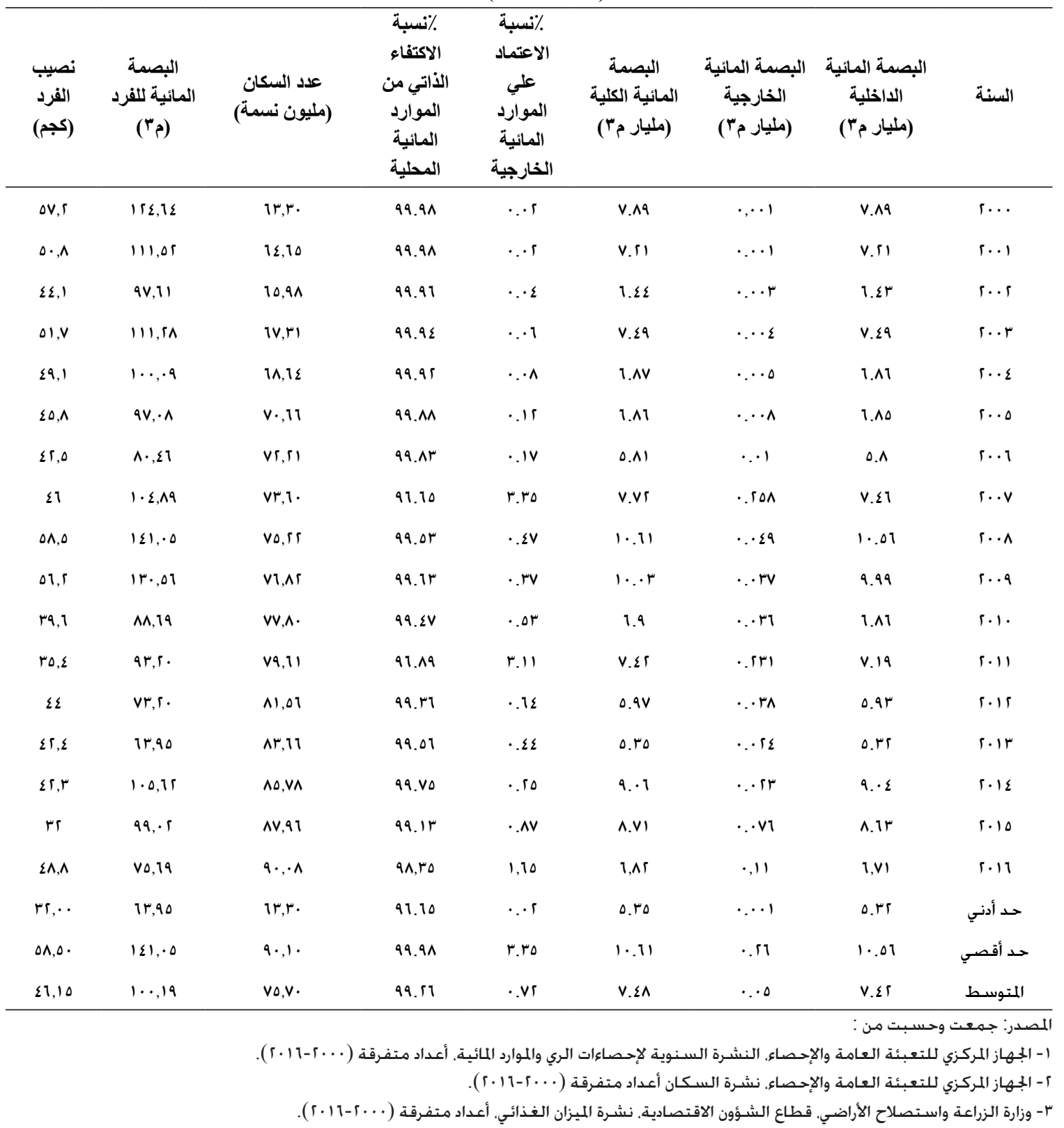

مجلة العلوم الزراعية المســدامة مهـ ، عـ (19 ؟) 


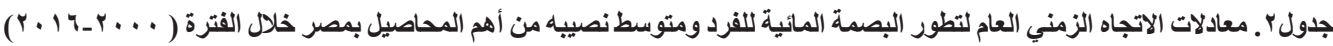

\begin{tabular}{|c|c|c|c|c|c|c|c|}
\hline معدل & ف ف & ז & المعادلة & النوع & الوحدة الو & البيان & المحصول \\
\hline r & ${ }^{*}(\Sigma, \Sigma \Gamma)$ & $\cdot$, ITA & 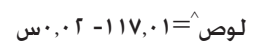 & أسي & $r_{p}$ & البصمة المائية للفرد & \\
\hline I,V & ${ }^{*}(\Delta, v r)$ & $\cdot$, IV & لوص=|l|r, & أسي & كجهم & متوسط نصيب الفرد & الأرز \\
\hline$\therefore$, vo & $-(1,9 \pi)$ & $\cdot, 11 \varepsilon$ & 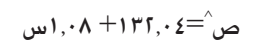 & خطي & $r_{p}$ & البصمة المائية للفرد & \\
\hline$r, 1 \wedge$ & $* *(r \Delta, v v)$ & $\cdot, \mathrm{V} \cdot \mathrm{o}$ & ص^=^ז = & خطي & كجه & متوسط نصيب الفرد & الذرة الشـامية \\
\hline$\varepsilon, r$ & ${ }^{* *}(\varepsilon \Delta, q 1)$ & $\cdot, \vee \vee \varepsilon$ & لوص = = لـ & أسي & $r_{p}$ & البصمة المائية للفرد & القمـح \\
\hline$\cdot r$ & $-\left(1, \sum v\right)$ & $\cdot, \cdot \wedge 9$ & 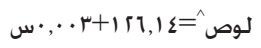 & أسي & كجهم & متوسط نصيب الفرد & \\
\hline
\end{tabular}

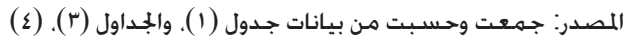

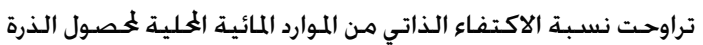

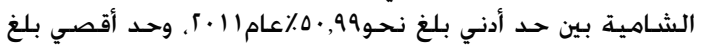

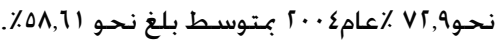

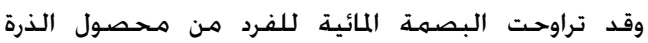

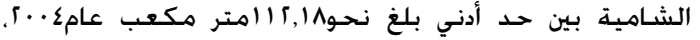

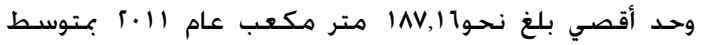

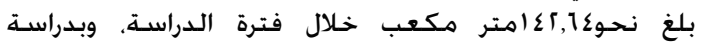

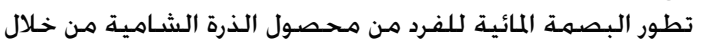

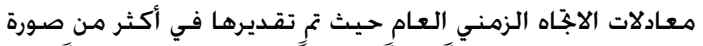

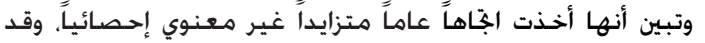

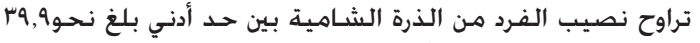

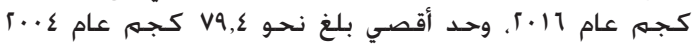

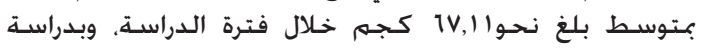

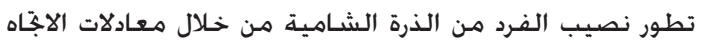

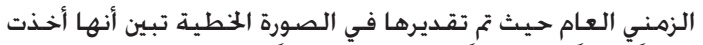

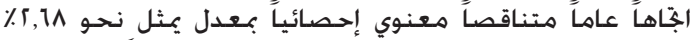

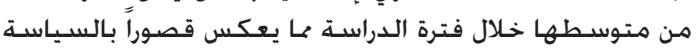

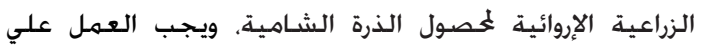

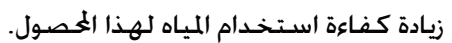

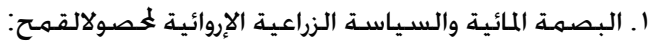

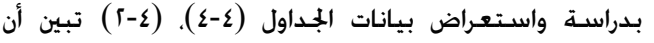

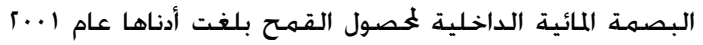

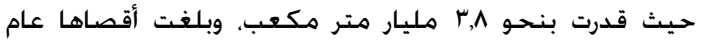

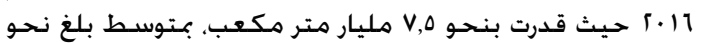

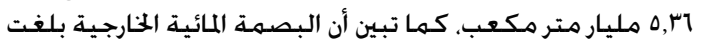

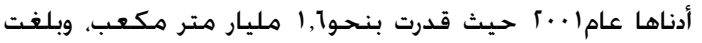

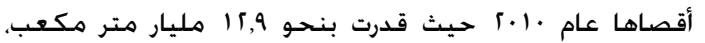

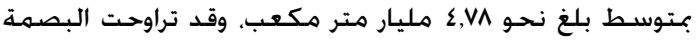

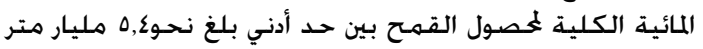

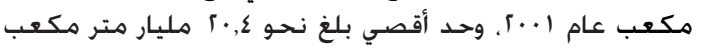

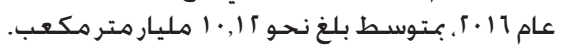

وقد تراوحت البصمة المائية للفرد من محصول محس الأرز بين

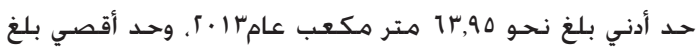

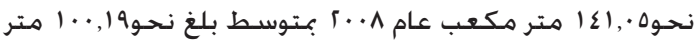

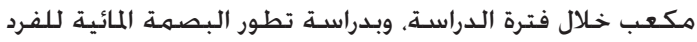

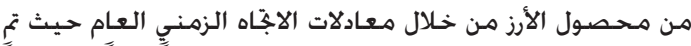

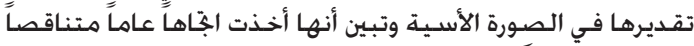

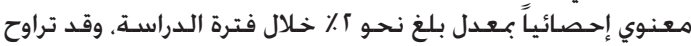

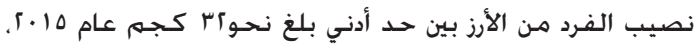

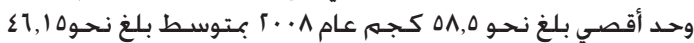

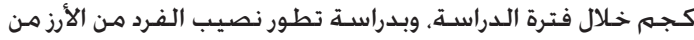

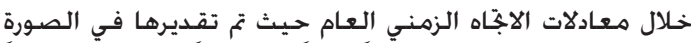

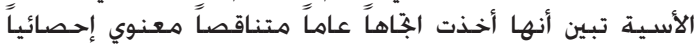

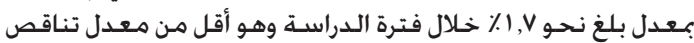

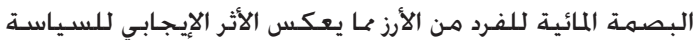
الزراعية الإروائية لمصول للفرد من الأرز

ז. البصمة المائية والسياسـة الزراعية الإروائية لغحصول الذرة

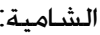

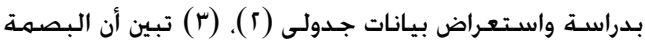

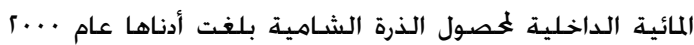

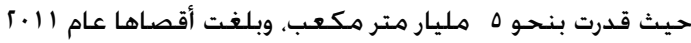

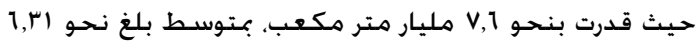

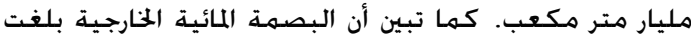

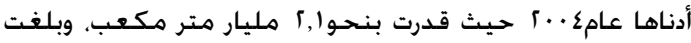

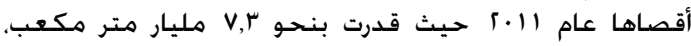

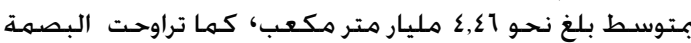

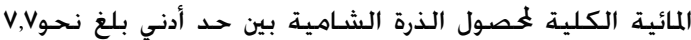

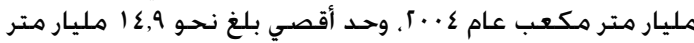

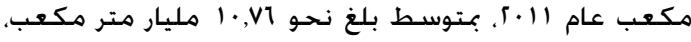

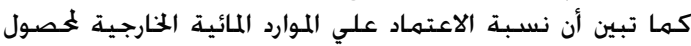

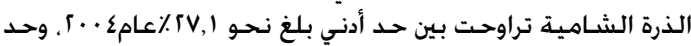

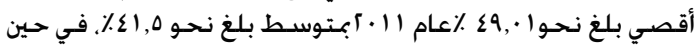




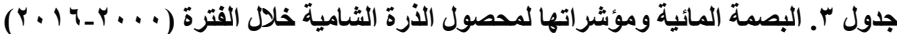

\begin{tabular}{|c|c|c|c|c|c|c|c|}
\hline نصيب الفرد & 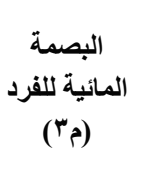 & نسبة الاكتفاء الذاتي & 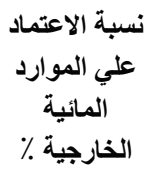 & 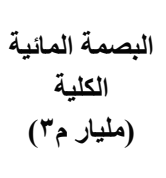 & البصمة المائية & البصمة المائية & السنة \\
\hline$v 1, r$ & $\mid r \varepsilon, \Gamma \Lambda$ & $\Delta \wedge, \wedge \Gamma$ & $\varepsilon 1,1 \wedge$ & $\Lambda, 0$ & $r, 0$ & 0 & $\lceil\ldots$ \\
\hline $\mathrm{v} 1, \varepsilon$ & $1 \leq 0, \varepsilon$. & $\Delta v, \varepsilon \varepsilon$ & $\left.\sum r, 0\right\}$ & $9, \varepsilon$ & $\varepsilon$ & $0, \varepsilon$ & $r \ldots 1$ \\
\hline$v V, v$ & $\mid \varepsilon r, \varepsilon V$ & $\Delta q, I V$ & $\varepsilon \cdot \wedge r$ & $9, \varepsilon$ & $r, q$ & 0,1 & $r \ldots r$ \\
\hline $\mathrm{vV}, \mathrm{A}$ & I IV V,VV & $11, \mathrm{VA}$ & rA, IT & $\Lambda, 1$ & $r, r$ & $0, r$ & $r . . r$ \\
\hline$v q, \varepsilon$ & $\| r, 1 \wedge$ & Vr,AM & rV, Ir & $v, v$ & $\Gamma, 1$ & 0,1 & $r \cdots \varepsilon$ \\
\hline $\mathrm{VV}, \mathrm{\Lambda}$ & $1 \leqslant \Gamma, 9 \varepsilon$ & $\Delta 1, \Lambda$ & $\varepsilon \mu, r$ & $1 \cdot, 1$ & $\varepsilon, \varepsilon$ & $\Delta, \wedge$ & r..o \\
\hline$v \wedge, 1$ & $\mid r \cdot, \Sigma \Lambda$ & IV, $\cdot 1$ & $r r, 99$ & $\Lambda, \vee$ & 5,9 & $0, \Lambda$ & $r . . r$ \\
\hline vi,v & $1 r \wedge, \Delta 9$ & $1 \cdot, 09$ & $r q, \Sigma 1$ & $1 \cdot, r$ & $\varepsilon$ & $1, r$ & $r \cdots v$ \\
\hline IV,o & $10 \Gamma, \Lambda \wedge$ & $\Delta v, v 1$ & $\varepsilon r, \Gamma q$ & 11,0 & $\varepsilon, \Lambda$ & 1,1 & $\Gamma \cdots \wedge$ \\
\hline $11, r$ & $1 \Sigma \Delta, \wedge$. & $V T, \cdot r$ & $r v, q \Lambda$ & $11, r$ & $\varepsilon, r$ & $v$ & $r \ldots q$ \\
\hline$v 1, V$ & $\mid \Sigma q, 1$. & $\Delta \wedge, 11$ & $\sum 1, \wedge 9$ & 11,1 & $\varepsilon, 9$ & $1, V$ & r.1. \\
\hline TV,r & $1 \wedge V, 11$ & $0 \cdot, 99$ & $\sum q, \cdot 1$ & $1 \varepsilon, 9$ & $v, r$ & $v, 1$ & {$[.11$} \\
\hline $1 \cdot, 9$ & IEr, r & 01,50 & $\Sigma \wedge, \vee \Delta$ & 11,1 & $\Delta, V$ & 0,9 & r.15 \\
\hline $10, \varepsilon$ & $\mid \varepsilon r, \Sigma \varepsilon$ & $\Delta \wedge, 11$ & $\sum 1, \wedge 9$ & 15 & 0 & $v$ & r.1r \\
\hline$\Delta \wedge, 1$ & $159, \varepsilon$. & $1 \varepsilon, \Delta \Lambda$ & $r \Delta, \varepsilon r$ & 11,1 & $r, q$ & $v, r$ & r.1E \\
\hline$\sum \wedge, 0$ & $10 \Delta, \vee \Delta$ & $\Delta\{, 11$ & $\Sigma \Delta, \wedge \varepsilon$ & $1 r, v$ & $1, r$ & $v, \varepsilon$ & 1.10 \\
\hline$r 9,9$ & $1 \varepsilon \cdot, 90$ & $\Delta 1, \cdot 1$ & $\varepsilon r, q \varepsilon$ & $15, \mathrm{~V}$ & 0,1 & $v, 1$ & 1.11 \\
\hline$r q, q$. & $\| r, 11$ & $0 \cdot, 99$ & IV, Ir & $\mathrm{v}, \mathrm{v}$. & $r, 1$. & $\Delta, \cdot \cdot$ & حـد أدني \\
\hline$v q, \varepsilon$. & $\mid \wedge V, 11$ & $\mathrm{Vr}, \Lambda \mathrm{A}$ & $\sum 9, \cdot 1$ & $1 \varepsilon, q$. & $v, r$. & $\mathrm{v}, \mathrm{r}$. & حد أقصي \\
\hline $\mid \mathrm{V}, 11$ & $1 \varepsilon r, 1 \varepsilon$ & $\Delta \wedge, 11$ & $\Sigma 1,0$. & $1 \cdot, \mathrm{V} 1$ & $\varepsilon, \Sigma\rceil$ & $1, r 1$ & المتوسطط \\
\hline
\end{tabular}

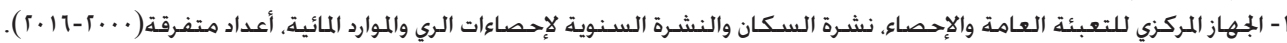

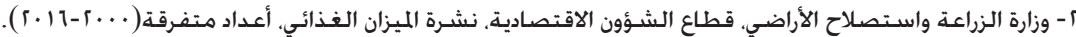

ثانياً: جنارة المياه الافتراضية لأهم محاصيل الحبوب:

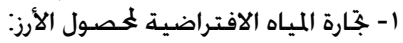

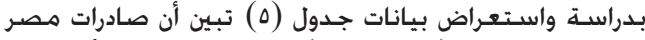

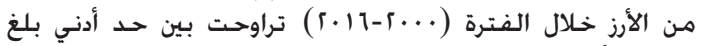

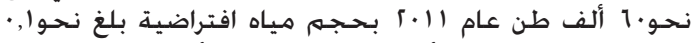

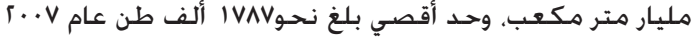

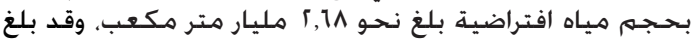

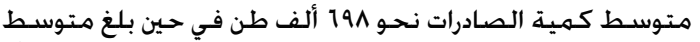

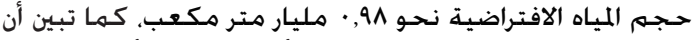

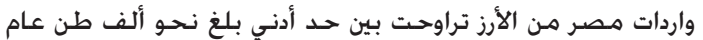

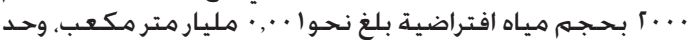

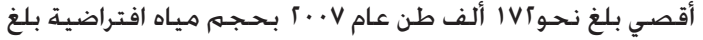

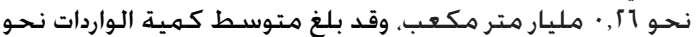

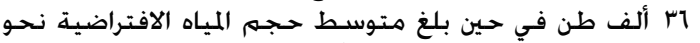
0

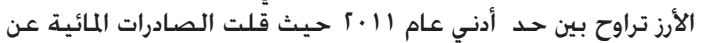

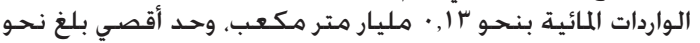

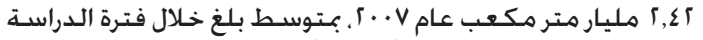

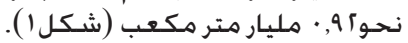

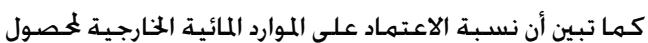

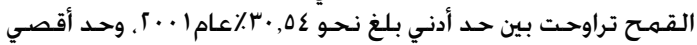

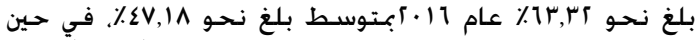

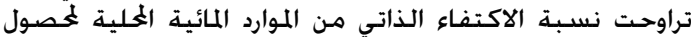

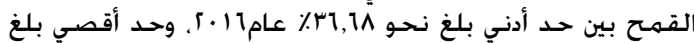

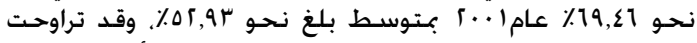

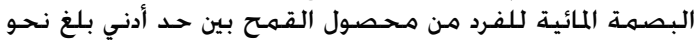

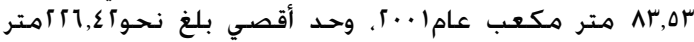

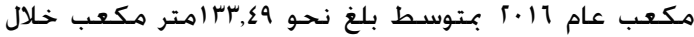

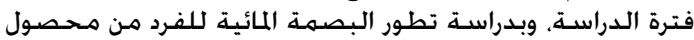

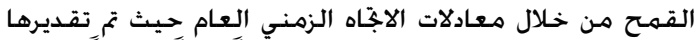

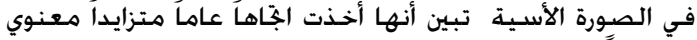

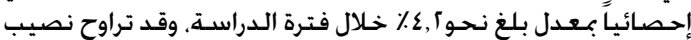

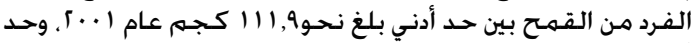

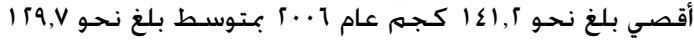

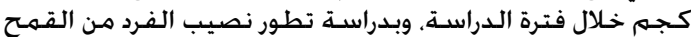

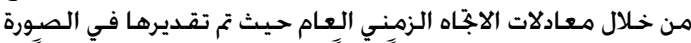

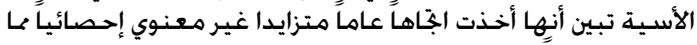

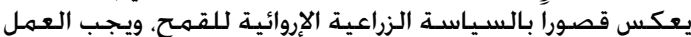

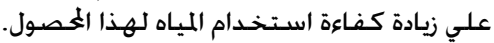




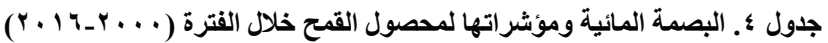

\begin{tabular}{|c|c|c|c|c|c|c|c|}
\hline نصيب الفرد & اللفرد (مّ) & $\begin{array}{c}\text { المبة الاكتي من الماءد المائية } \\
\text { المحلية } \\
\text { \% }\end{array}$ & $\begin{array}{c}\text { نسبة الاعتماد الموارد } \\
\text { الخارجية }\end{array}$ & البصمة المائية & البصمة المائية & البصمة المائية & السنة \\
\hline 159,1 & $1 \cdot r, 19$ & $1 \cdot, \cdot 1$ & $r 9,99$ & 1,0 & $r, 1$ & $r, q$ & $r \ldots$ \\
\hline 111,9 & $\Delta r, \Delta r$ & $19, \Sigma 1$ & $r \cdot, \Delta \varepsilon$ & $0, \varepsilon$ & 1,1 & $r, \Lambda$ & $\lceil. .1$ \\
\hline $1 \Gamma \Lambda$ & $1.1,00$ & $\Delta \wedge, \vee$ & $\varepsilon 1, r$ & $\mathrm{I}, \mathrm{V}$ & $\Gamma, \Lambda$ & $r, q$ & $r \ldots r$ \\
\hline$\|\lceil\|, \varepsilon$ & $৭ ৭, \Delta \varepsilon$ & $\mid r, \wedge 1$ & $r v, 19$ & $\mathrm{~T}, \mathrm{~V}$ & $r, 0$ & $\varepsilon, r$ & $r . . r$ \\
\hline IrV,r & $1.1,91$ & Ir,IV & $r v, \wedge r$ & $v$ & $r, V$ & $\varepsilon, \varepsilon$ & $\Gamma \cdots \varepsilon$ \\
\hline 1ro. & $\| 11, \wedge$. & $\Delta \wedge, \wedge \vee$ & $\varepsilon 1,1 \pi$ & $v, q$ & $r, r$ & $\varepsilon, V$ & $r \ldots \Delta$ \\
\hline$|\varepsilon|, \Gamma$ & $|r|, 01$ & $\Delta \wedge, \vee \Delta$ & $\sum 1,50$ & 9,0 & $r, 9$ & 0,1 & $r . .1$ \\
\hline 1 irr, 9 & $1[\pi, 1 \varepsilon$ & $\Delta \Delta, \Delta \varepsilon$ & $\varepsilon \varepsilon, \varepsilon 1$ & 9,1 & $\varepsilon, 1$ & 0,1 & $r \cdots v$ \\
\hline $1 \pi 1,1$ & 174,09 & 01,90 & $\sum \Lambda, \cdot \Delta$ & $1 \cdot, 0$ & 0 & $0, \varepsilon$ & $\Gamma \cdots \wedge$ \\
\hline $1 r 0, \varepsilon$ & $\mid \Gamma \varepsilon, q V$ & $\Delta \Delta,[1$ & $\varepsilon \varepsilon, \vee q$ & 9,1 & $\varepsilon, r$ & $0, r$ & $r . . q$ \\
\hline 1 rreq & 171,90 & $\varepsilon \Gamma, \Gamma \mu$ & $\Delta V, V V$ & 15,1 & $v, r$ & $0, r$ & r.l. \\
\hline 1ro,. & $1 \mu q, \varepsilon r$ & $\sum 1, \cdot 1$ & $\Delta r, q \varepsilon$ & 11,1 & 1 & 0,1 & {$[.11$} \\
\hline$|\Gamma|, V$ & 110,50 & $\Delta V, \varepsilon r$ & $\sum \Gamma, \Delta V$ & $9, \varepsilon$ & $\varepsilon$ & $0, \varepsilon$ & $r .1 r$ \\
\hline וrr,r & $1 \leq \Delta, \Lambda \mu$ & $\Delta \Lambda, \Gamma \mu$ & $\varepsilon I, V V$ & 18,5 & 0,1 & $\mathrm{v}, 1$ & $r .1 r$ \\
\hline $15 \mathrm{~V}, 0$ & $\mid \Delta 9, V 1$ & גז,rם & $\varepsilon 1,1 \Gamma$ & $1 r, v$ & $1, \varepsilon$ & $v, r$ & r.1E \\
\hline $\mid[\Lambda, \Lambda$ & $107, \wedge 9$ & & $\sum \Lambda, r \Gamma$ & $1 \%, \Lambda$ & $\mathrm{T}, \mathrm{V}$ & $v, 1$ & 1.10 \\
\hline irr. & {$[\lceil T, \varepsilon r$} & & זr, זr & $\Gamma \cdot, \varepsilon \cdot$ & 15,9 & $\mathrm{v}, \mathrm{o}$. & 1.11 \\
\hline 111,9 & $\Delta r, \Delta r$ & $r 1,1 \wedge$ & $r \cdot, 0 \varepsilon$ & $0, \varepsilon$. & 1,1 & $r, \wedge$. & حـد أدني \\
\hline$|\varepsilon|, \Gamma$ & $\Gamma[T, \varepsilon \Gamma$ & $19, \Sigma 1$ & זr, & $\Gamma \cdot, \Sigma \cdot$ & $15,9$. & $\mathrm{v}, \mathrm{o}$. & حد أقصي \\
\hline 159,8 & Irr, & $\Delta \Gamma, Q T$ & $\sum V, 1 \Lambda$ & $1 \cdot, 15$ & $\varepsilon, \mathrm{VA}$ & דוז & المتوسـط \\
\hline
\end{tabular}

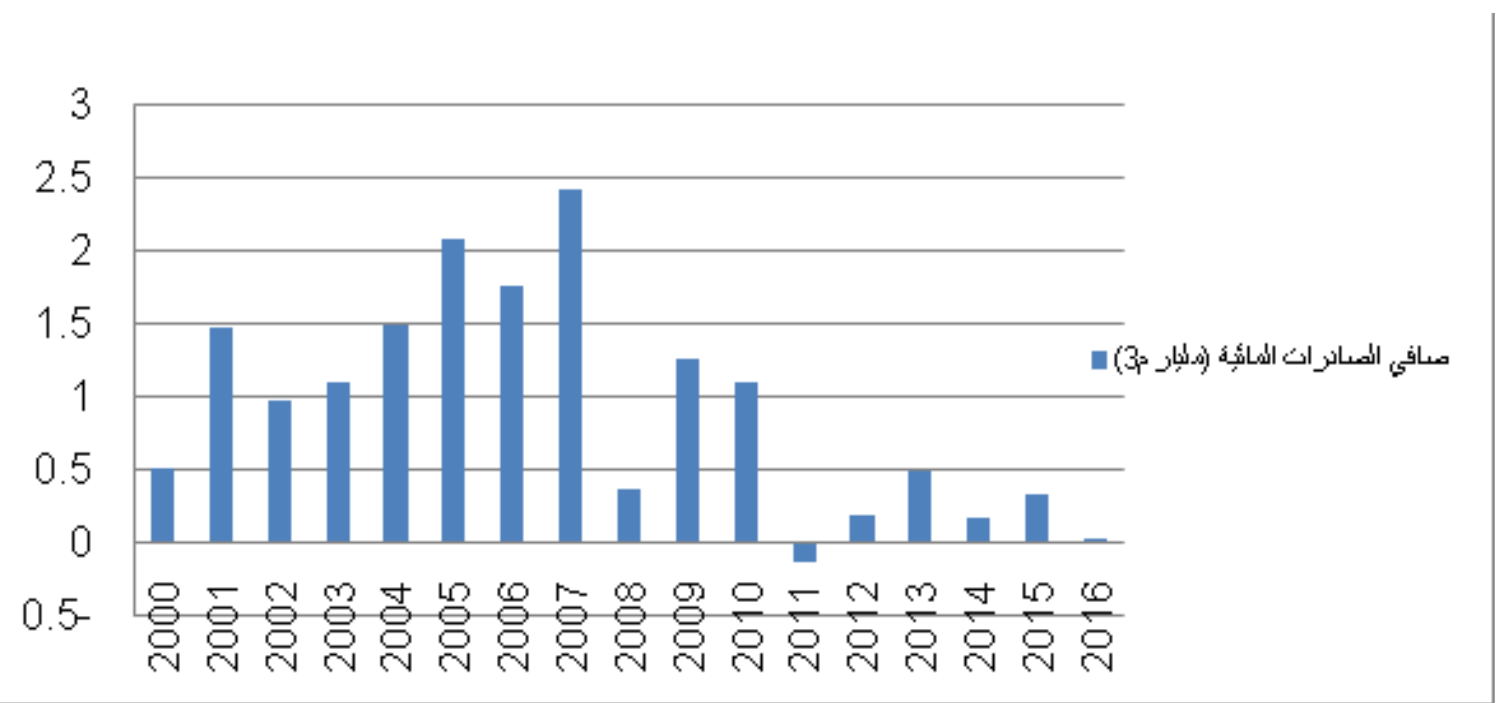

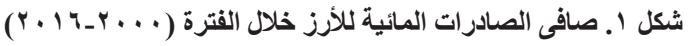

مجلة العلوم الزراعية المستدامة مع ،عـ (19 • ) ) 


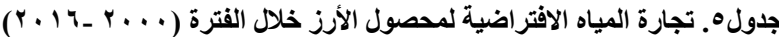

\begin{tabular}{|c|c|c|c|c|c|}
\hline \multirow{2}{*}{ صافي الصادرات } & \multicolumn{2}{|c|}{ واردات } & \multicolumn{2}{|c|}{ صادرات } & \multirow[b]{2}{*}{ السنة } \\
\hline & مليار مج & ألف طن & مليار مץ & ألف طن & \\
\hline .019 & $\because \cdots 1$ & 1 & $\cdot, \Delta r$ & $r q$. & $r \ldots$ \\
\hline 1.29 & $\because \cdots 1$ & 1 & $1, \Sigma 9$ & $1 . \mu$. & $\lceil\ldots 1$ \\
\hline. .99 & $\because . r r$ & r & $\cdot, 99$ & 191 & $r \ldots r$ \\
\hline 1.1 & $\because \cdots \varepsilon$ & $r$ & 1,1 & VVq & $r . . r$ \\
\hline 1.0 & $\because \cdots \Delta$ & $\varepsilon$ & 1,0 & 111. & $r \cdots \varepsilon$ \\
\hline $5 . .9$ & $\because \cdots \wedge$ & 1 & $\Gamma, 1$ & $1 \leqslant \wedge q$ & $r \cdots \Delta$ \\
\hline $1 . v 1$ & $\because \cdot 1$ & $\wedge$ & I,VV & $1 \leq r \Delta$ & 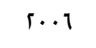 \\
\hline r.25 &. .11 & IVI & ז, & IVAV & $r \ldots v$ \\
\hline. $\mathrm{rv}$ & $\because \varepsilon q 9$ & $r 1$ & $\cdot, \Sigma \Gamma$ & [11 & $r \cdots \wedge$ \\
\hline 1.11 & $\because r v$ & is & $1, r$ & ה & $r \ldots q$ \\
\hline 1.11 & 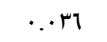 & 10 & 1,10 & V9o & r.1. \\
\hline$(\cdot .1 r)$ & וזr. & Irv & $\cdot, 1$ & 1. & {$[.11$} \\
\hline.$r$ & $\cdot \cdot r \Lambda$ & ro & $\cdot, \Gamma \varepsilon$ & rro & r.1r \\
\hline .0 & $\because \cdot r \varepsilon$ & rs & •, Or & 019 & $r .1 T$ \\
\hline. $.1 \mathrm{~V}$ & . & $1 \varepsilon$ & $\cdot, 19$ & 15. & $r .1 \varepsilon$ \\
\hline.$r \varepsilon$ & $\because \cdot \mathrm{v}^{1}$ & $\varepsilon 1$ & $\cdot, \sum \Gamma$ & ror & 1.10 \\
\hline$\cdot, \cdot r$ & $\cdot, 11$ & va & $\cdot, 1 \varepsilon$ & $1 \ldots$ & $r .17$ \\
\hline$(\cdot .1 \%)$ & $\cdot, \cdot$ & $1, \cdot \cdot$ & $\cdot, 1$. & $1 \cdot, \cdot \cdot$ & حـد أدني \\
\hline$\Gamma, \Sigma \Gamma$ & ד & ivr,.. & $\Gamma, 1 \wedge$ & $I V \wedge v, \cdot \cdot$ & حدأقصي \\
\hline$\cdot, 95$ & $\cdot, \cdot 0$ & $r 1, \cdot \cdot$ & $\cdot, 91$ & $19 \Lambda, \cdot \cdot$ & المتوسط \\
\hline
\end{tabular}

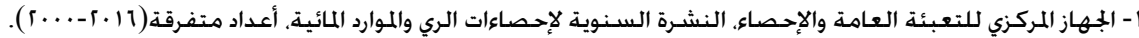

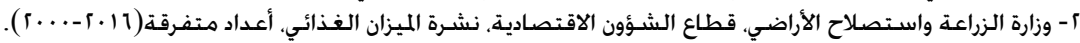

نحـو 199 امليون متر مكعب، وقد بلغ متوسـ كميـة الصادرات

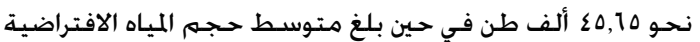

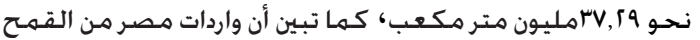

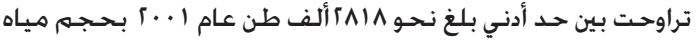

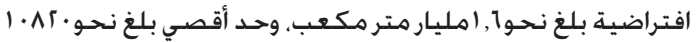

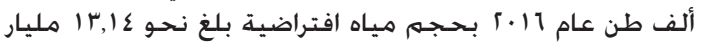

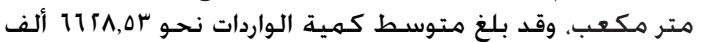

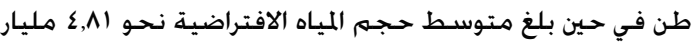

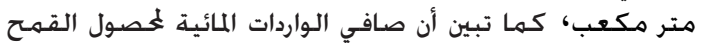

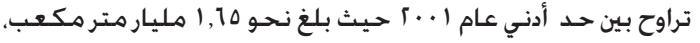

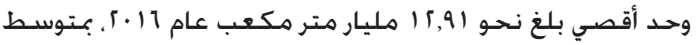

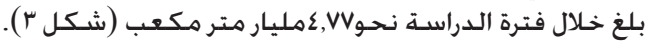

$$
\text { وقد توصلت الدراسـة إلي ما يلي: }
$$

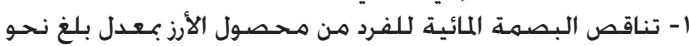

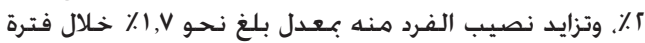

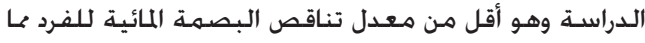

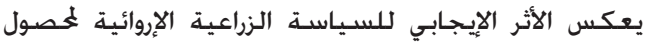

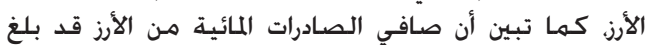

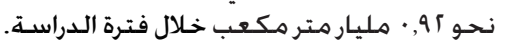

أ- تزايد البصمة المائية للفرد من الذرة الشـامية بصـورة غير

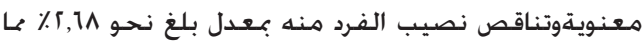

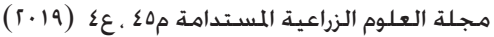

ا - جبّارة المياه الافتراضية لحُصـول الذرة الشـامية:

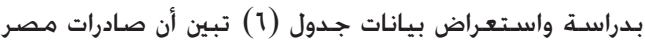

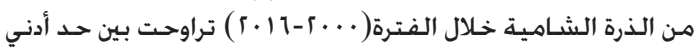

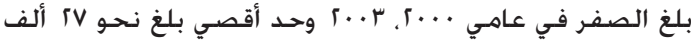

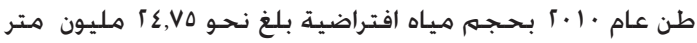

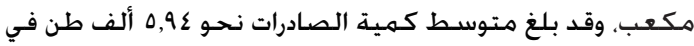

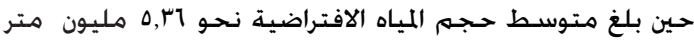

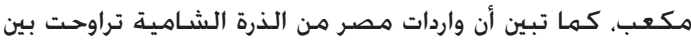

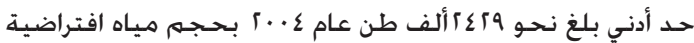

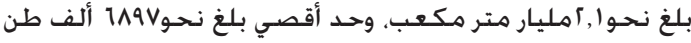

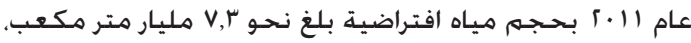

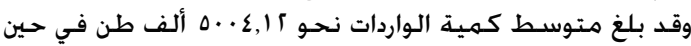

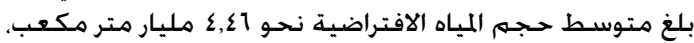

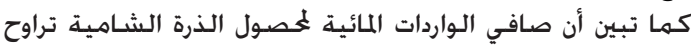

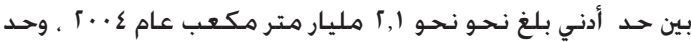

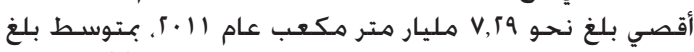

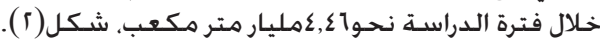

ب- جَّارة المياه الافتراضية لمحسول القـمـح:

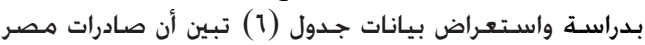

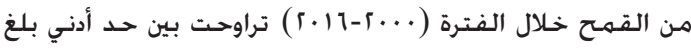

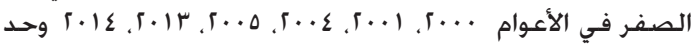

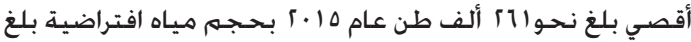




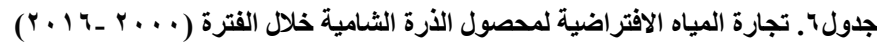

\begin{tabular}{|c|c|c|c|c|c|}
\hline \multirow{2}{*}{ صافي الواردات } & \multicolumn{2}{|c|}{ واردات } & \multicolumn{2}{|c|}{ صادرات } & \multirow[b]{2}{*}{ السنة } \\
\hline & مليار مج & ألف طن & مليون مج & ألف طن & \\
\hline$r, 0$. & $r, 0$. & $\varepsilon r \cdot r$ & $\cdot, \cdot$ & $\cdot, \cdot$ & $\ulcorner\ldots$ \\
\hline$\varepsilon, \cdot \cdot$ & $\varepsilon, \cdot \cdot$ & EVaV & . Ar & $1, \cdot \cdot$ & 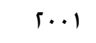 \\
\hline$r, q$. & $r, q$. & EVrl & ז', & $1, \cdot \cdot$ & r..r \\
\hline$r, r$. & $r, r \cdot$ & ravi & $\cdot, \cdot \cdot$ & $\cdot, \cdot$ & $r \ldots r$ \\
\hline r, . & $r, 1$. & rera & I,Vr & $r, \cdot$. & $r \cdots \varepsilon$ \\
\hline$\varepsilon, \varepsilon$. & $\varepsilon, \varepsilon$. & $011 \pi$ & $\varepsilon, \Gamma \Lambda$ & $0, \cdot \cdot$ & $r \cdots \Delta$ \\
\hline$r, q$. & $r, q$. & rVAM & r,vV & $0, \cdot \cdot$ & $\lceil\cdots \uparrow$ \\
\hline$r, 99$ & $\varepsilon, \cdot \cdot$ & $\varepsilon \varepsilon q$. & 1,59 & $\mathrm{v}, \cdot \cdot$ & $r \cdots v$ \\
\hline$\varepsilon, \Lambda$. & $\varepsilon, \Lambda$. & $0 . v \Delta$ & $\varepsilon, V \wedge$ & $0, \cdot \cdot$ & $\uparrow \cdots \wedge$ \\
\hline$\varepsilon, 19$ & $\varepsilon, r$. & sorv & $v, 0 \varepsilon$ & $\Lambda, \cdot \cdot$ & $r \ldots q$ \\
\hline$\varepsilon, \wedge \wedge$ & $\varepsilon, q$. & $\Delta r \cdot \varepsilon$ & $r \varepsilon, v \Delta$ & $i v, \cdot$. & $\lceil\cdot 1$. \\
\hline$v, 59$ & $v, r \cdot$ & $1 \wedge 9 \mathrm{~V}$ & $1, r o$ & $1, \cdot \cdot$ & $\lceil .11$ \\
\hline 0,11 & $0, v$. & 1015 & 10,11 & $1 \wedge, \cdot \cdot$ & r.1r \\
\hline $0, \cdot \cdot$ & $0, \cdot \cdot$ & DArr & I,vr & $r, \cdot \cdot$ & $\Gamma .1 \%$ \\
\hline$r, q$. & $r, q$. & $\varepsilon r u$ & $\varepsilon, \Delta r$ & $0, \cdot \cdot$ & $\Gamma .1 \varepsilon$ \\
\hline $1, r$. & $1, r$ & IAT. & $\ulcorner, \mathrm{V}\rceil$ & $r, \cdot \cdot$ & 1.10 \\
\hline 0,00 & 0,1 . & 1115 & $0, \Sigma 1$ & $1, \cdot \cdot$ & 1.11 \\
\hline$r, 1$. & $r, 1$. & $1 \sum 19$ & $\cdot, \cdot$ & $\cdot, \cdot$ & حـد أدني \\
\hline$v, 19$ & $v, r$. & $1 \wedge 9 \mathrm{~V}$ & $i \varepsilon, V \Delta$ & $i v, \cdot \cdot$ & حـدأقصي \\
\hline$\varepsilon, \varepsilon 1$ & $\varepsilon, \Sigma 1$ & $0 \cdot \varepsilon, 15$ & $0, r 1$ & $0,9 \varepsilon$ & لمتوسـط \\
\hline
\end{tabular}

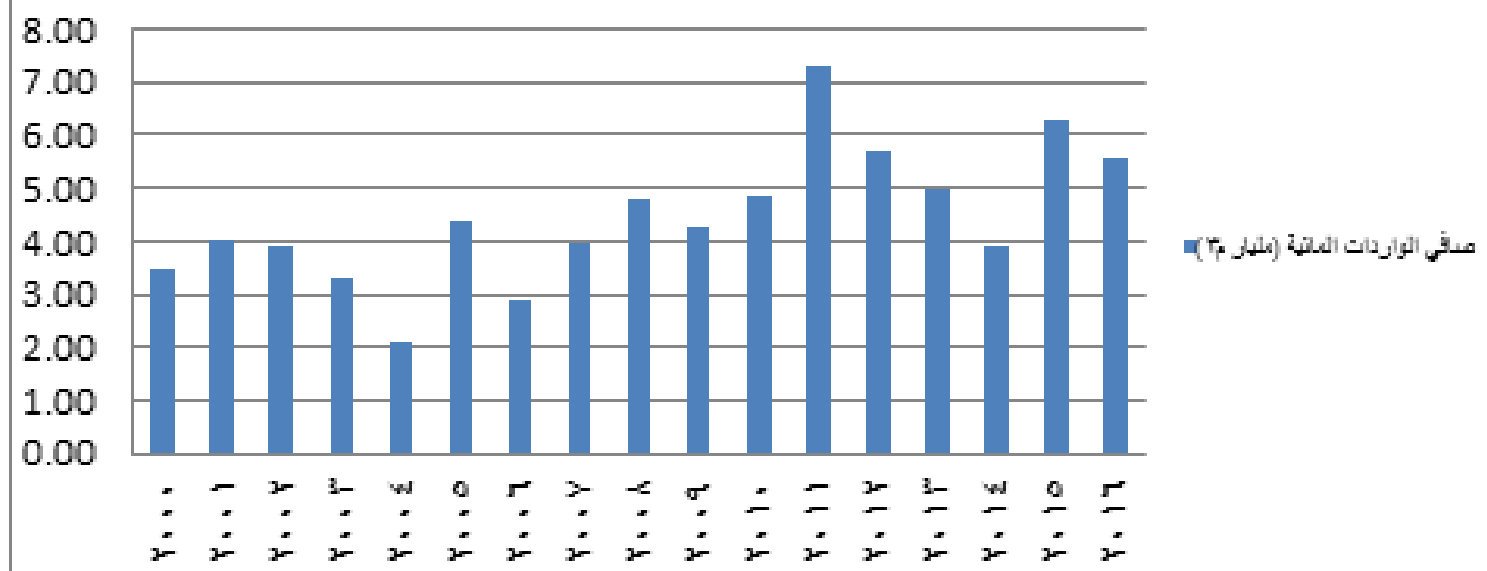

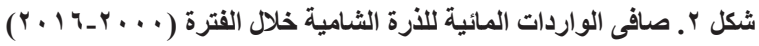

مجلة العلوم الزراعية المستدامة مع ،عـ (19 • ) ) 


\begin{tabular}{|c|c|c|c|c|c|}
\hline \multirow{2}{*}{ صافي الواردات } & \multicolumn{2}{|c|}{ واردات } & \multicolumn{2}{|c|}{ صادرات } & \multirow[b]{2}{*}{ السنة } \\
\hline & مليار مج & ألف طن & مليون مجّ & ألف طن & \\
\hline ז,1ז & $r, 1$ & $\varepsilon r \cdot r$ & . & . & $\ulcorner\ldots$ \\
\hline 1,10 & 1,1 & {$[A \mid \Lambda$} & . & . & 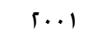 \\
\hline r,VV & $\ulcorner, \wedge$ & EOrl & r & $r$ & $r \cdots r$ \\
\hline$\Gamma, \Sigma \wedge$ & r,o & $2 \cdot 10$ & 11 & 19 & $r . . r$ \\
\hline$\ulcorner, 11$ & 「,V & $\varepsilon r T V$ & . & $\cdot$ & $r \cdots \varepsilon$ \\
\hline r, ro & $r, r$ & $\Delta 1 \wedge \Lambda$ & . & . & $r \cdots \Delta$ \\
\hline$r, 9 r$ & $r, 9$ & $\Delta \wedge r$. & is & ro & 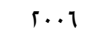 \\
\hline$\varepsilon, \cdot 1$ & $\varepsilon, 1$ & $\Delta 911$ & $1 \varepsilon$ & 11 & $r \cdots v$ \\
\hline $0, \cdot r$ & $0, \cdot$ & vrAl & 10 & it & $\lceil\cdots \wedge$ \\
\hline$\varepsilon, \Gamma \wedge$ & $\varepsilon, r$ & $19 \pi r$ & 11 & $9 v$ & $r \cdots q$ \\
\hline$v, \Gamma v$ & $v, r$ & 91.0 & $\varepsilon$ & 0 & r. \\
\hline $0,9 \mathrm{~V}$ & $1, \cdot$ & $9 \Lambda \cdot \Sigma$ & r & $\varepsilon$ & {$[\cdot 11$} \\
\hline$\varepsilon, \cdot r$ & $\varepsilon, 1$ & $10 \leq 9$ & v) & $11 \varepsilon$ & $r .15$ \\
\hline $0, .9$ & 0,1 & IVAs & . & . & $r .1 \%$ \\
\hline $1, \mathrm{rV}$ & $1, \varepsilon$ & $11 \cdot 0$ & $\cdot$ & $\cdot$ & $1.1 \varepsilon$ \\
\hline $1,1 \mathrm{~V}$ & 1,9 & $9 . .1$ & 199 & [11 & 1.10 \\
\hline 15,91 & $1 \pi, 1 \varepsilon$ & $1 \cdot \wedge r$. & ris & InD & 1.17 \\
\hline 1,10 & 1,1 & [AIA & . & . & حد أدني \\
\hline$|r, 9|$ & $1 r, 1 \varepsilon$ & $1 \cdot \wedge r$. & irs & ז11 & حدأقصي \\
\hline$\varepsilon, \mathrm{VV}$ & $\varepsilon, \wedge 1$ & $11 \Gamma \Lambda, \Delta H$ & $r V, r q$ & $\varepsilon 0,10$ & المتوسط \\
\hline
\end{tabular}

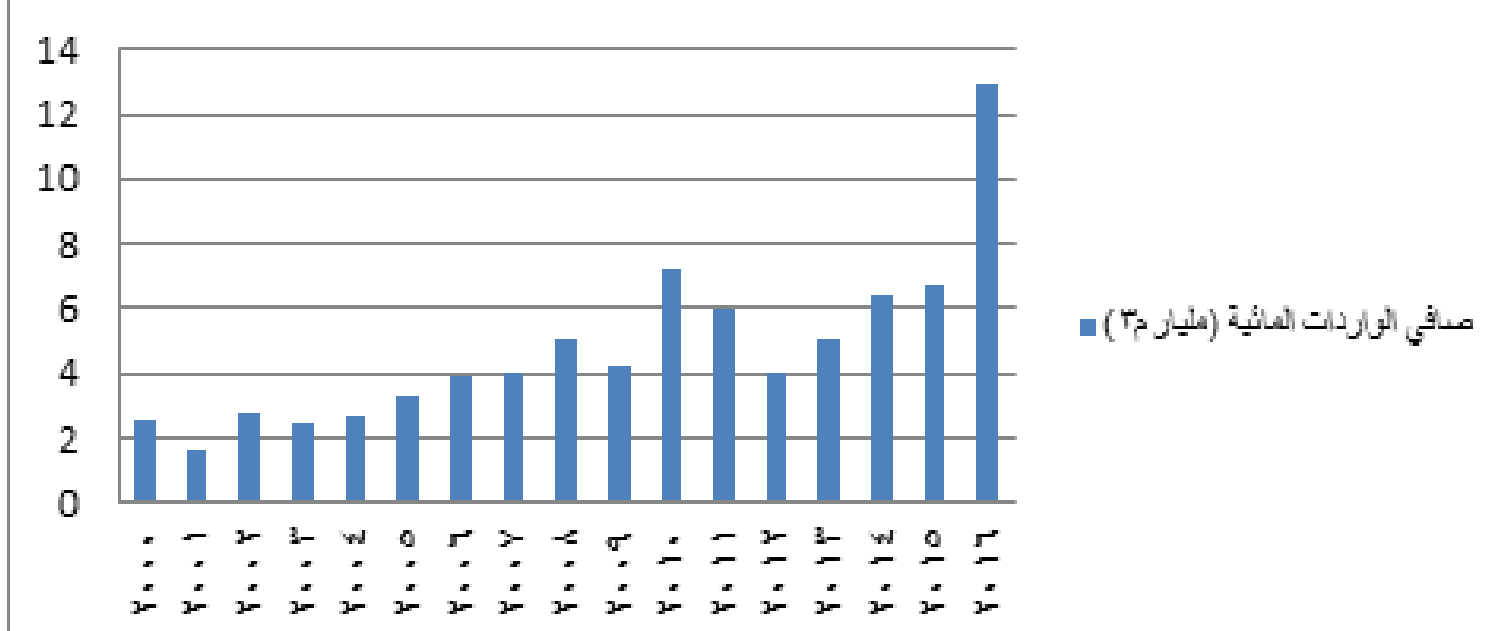

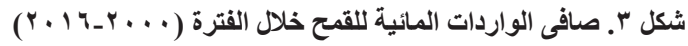

مجلة العلوم الزراعية المستدامة مـع .عـ (19 • م) 
^- الجهاز المركزي للتعبئة العامة والاحصاء، النشـرة السنوية

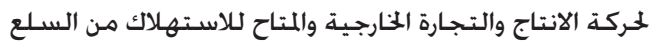

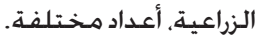

9- الجهاز المركزي للتعبئة العامة والإحصاء، نشـرة احصاءات البيئة، أعداد متفرقة.

• ر- قمره، سـحر عبد المنعم السيد: تقدير البصمة المائية والمياه

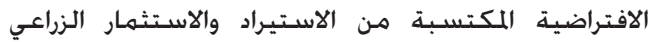

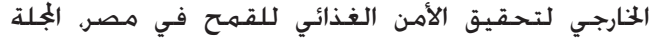

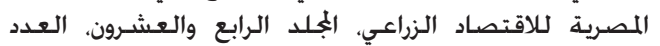

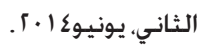

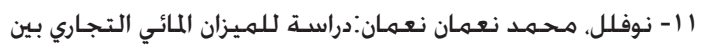

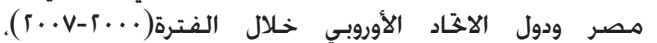

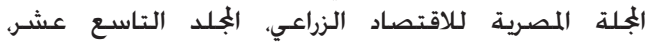

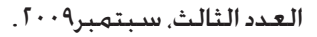

أ ا- عرفه، محمود عبدالتواب: دراسـة خليلية للاستخــام الأمثل

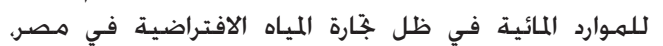

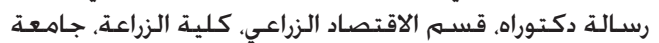

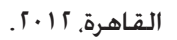

" ا - سيد، مهاعبدالفتاح ابراهيهم:دراسـة اقتصادية للتجارة الزراعية

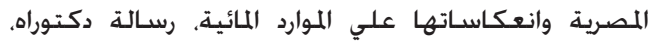
قسـم الاقتصاد الزراعي، كلية الزراعة، جامعة عين شـمس. .1 .9

عا- محسمدولاء حسين عبدالله:المياه الافتراضية وأثرها علي

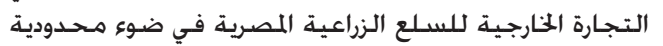

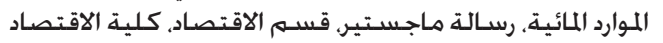

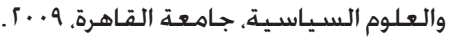

$$
\text { ثانياً: المراجع الأجنبية }
$$

1. Heady Earl,O., Economics of Agricultural Production and Resource use, New-york, N.Y., Prentice-Hall.Inc,Englewood cliffs, N.J.1960.

2. Heady Earl,O. and Dillion J.L., Agricultural Production Function, Ames, Iawa,TheIawa state university, press, 1961..

ثالثاً: مواقع شبكة المعلومات الدولية:

1. www.campas.gov.eg

2. www.waterfootprint.org

3. www.worldbank.org
يعكس قصوراً بالسياسـة الزراعية الإروائية للذرة الشـامية،

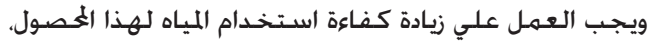

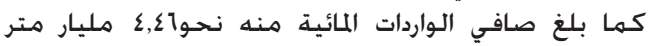
مكعب خلال فترة الدراسـة.

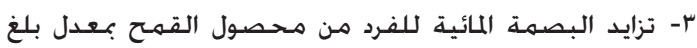

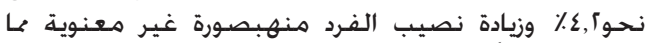

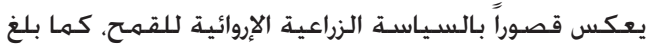

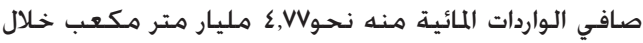

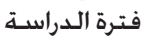

ويوصي البحث بما يلي:

1- ضرورة حساب البصمة المائية لخختلف الخحاصيل للوصول

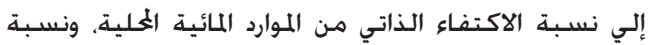
الاعتماد علي الموارد المائية الخارجية.

ז- ضرورة معالجة أوجه القصور بالسياسـة الزراعية الإروائية

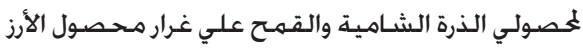

س- الاهتمام بمفهومي البصمة المائيةوالمياه الافتراضية بما يحقق لئق

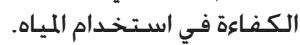

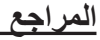
أولاً: المراجع باللغة العربية:

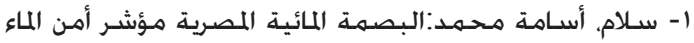

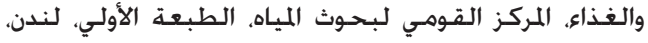

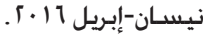

ז- عويضة ، أسـامة محممود ، السيد السيد جاد عبدالرحمن

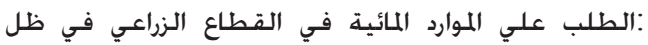

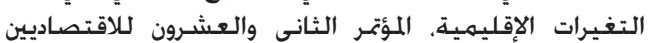

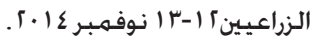

ץ- استراتيجية التنمية الزراعية المستدامة حتي عام ·r • ؟. ع- حماد ، أكرم محسمد محمـود: اقتصاديات استخخدام الموارد

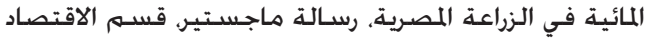

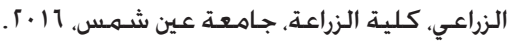

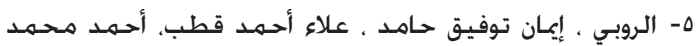

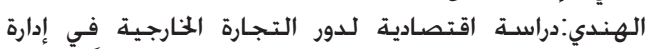

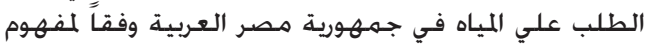

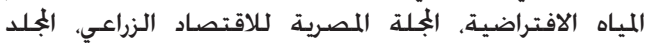

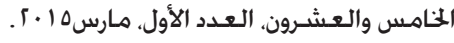

1- التقرير السـوي للمنتدي العربي للبيئة والتنمية (آفد) 1 1 ـ ؟.

V- الجهاز المركزي للتعبئة العامة والاحصاء، التقرير السنوي

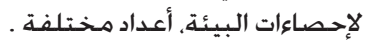




\title{
An Economic Study of The water Footprint and Virtual Water Trade of The Most Important Grain Crops in Egypt
}

Fawzi Mohammed El-Danasouri, Mahmoud Mohammed Fawaz, Ahmed Bedair El-Saadi* and Mohamed Ashraf Abd el Malek*

Agricultural Economics Branch, Agricultural Economics Department, Faculty of

Agriculture, Kafr El-Sheikh University, Egypt

*Agricultural Economics Research Institute - Agricultural Research Center, Egypt

\begin{abstract}
$\mathrm{N}$ VIEW of the current situation and the study of the water crisis experienced by Egypt it is important to develop new concepts to rationalize water consumption, including the concept of water footprint to estimate the volume of water needed to produce the various commodities consumed by humans, Cereal crops in Egypt, rice, maize and wheat during the period (20002016), the research was based on secondary data, and the use of both descriptive and quantitative economic analysis methods As long as the general time trend equations, the water footprint estimation model and its indicators, The most important results were a decrease in the per capita water footprint of the rice crop by about $2 \%$, and its per capita growth increased by about $1.7 \%$ during the study period, reflecting the positive impact of the agricultural irrigation policy of rice crop, while showing the increase of per capita water footprint of maize and a decrease in The per capita by about $2.68 \%$ and the per capita share of wheat increased by $4.2 \%$ and the per capita growth increased non significantly, reflecting a shortage of maize and wheat irrigation policy, The research recommends the need to calculate the water footprint of various crops, and recommends the need to address the shortcomings agricultural irrigation policy for maize and wheat crops, such as rice crop.
\end{abstract}

Key words: Water footprint, Average per capita yield, Self-sufficiency ratio of water resources 